\title{
Detection of single photons with THickGEM-based counters
}

\author{
M. Alexeev ${ }^{a}$, F. Barbosa ${ }^{k}$, R. Birsa ${ }^{j}$, F. Bradamante ${ }^{i}$, A. Bressan ${ }^{i}$, M. Chiosso ${ }^{g}$, P. Ciliberti ${ }^{i}$, S. Dalla Torre $^{j}$,

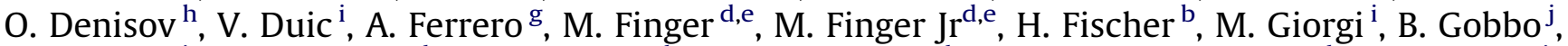 \\ M. Gregori ${ }^{j}$, F.H. Heinsius ${ }^{\text {b }}$, F. Herrmann ${ }^{b}$, K. Königsmann ${ }^{b}$, D. Kramer ${ }^{c}$, L. Lauser ${ }^{b}$, S. Levorato ${ }^{i, *}$,

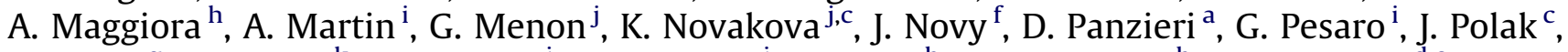

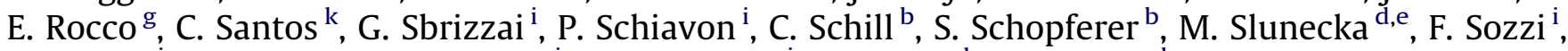 \\ L. Steiger $^{\mathrm{i}, \mathrm{c}}$, M. Sulc ${ }^{\mathrm{c}}, \mathrm{S}$. Takekawa ${ }^{\mathrm{i}}$, F. Tessarotto $^{\mathrm{j}}$, C. Veloso $^{\mathrm{k}}$, H. Wollny $^{\mathrm{b}}$
}

a INFN, Sezione di Torino and University of East Piemonte, Alessandria, Italy

b Universität Freiburg, Physikalisches Institut, Freiburg, Germany

c Technical University of Liberec, Liberec, Czech Republic

d Charles University, Praga, Czech Republic

e JINR, Dubna, Russia

${ }^{\mathrm{f}}$ Faculty of Nuclear Sciences and Physical Engineering, Czech Technical University, Prague, Czech Republic

${ }^{\mathrm{g}}$ INFN, Sezione di Torino and University of Torino, Torino, Italy

h INFN, Sezione di Torino, Torino, Italy

${ }^{\mathrm{i}}$ INFN, Sezione di Trieste and University of Trieste, Trieste, Italy

j INFN, Sezione di Trieste, Trieste, Italy

${ }^{\mathrm{k}}$ I3N - Physics Department, University of Aveiro, Portugal

\section{A R T I C L E I N F O}

Available online 8 December 2011

Keywords:

THGEM

Single photon detection

RICH

CsI

Photon extraction

\begin{abstract}
A B S T R A C T
Cherenkov imaging counters requiring large photosensitive areas, the capability to stand high rates and to operate in magnetic field environments could benefit from the use of photon detectors based on THick Gaseous Electron Multiplier (THGEM) coupled to a solid state CsI photo-cathode.

A systematic study of the THGEM detector response as a function of its geometrical parameters and electrodes' applied voltage has been performed. Dedicated electrostatic calculations to optimize the detector design have been accomplished. Data obtained from small photon detector prototypes operating in single photon detection mode are presented and discussed. In particular the key aspect of photo-electron extraction from the photo-cathode surface is investigated via the timing spectrum response of the detector for different electric field conditions at the photo-cathode: a comparison of the measured time distributions and the simulation results is illustrated.
\end{abstract}

(c) 2011 Elsevier B.V. All rights reserved.

\section{THGEM electron multipliers}

The THGEM [1,2] is a robust gaseous electron multiplier based on GEM principle scaling the geometrical parameters. It is obtained via standard PCB drilling and etching processes making possible the economic production of large series and large size devices. THGEM geometrical parameters cover wide ranges; typical values are as follows: $\mathrm{PCB}$ thickness from 0.3 to $1 \mathrm{~mm}$; holes diameter from 0.2 to $1.0 \mathrm{~mm}$; hole pitch from 0.4 to $1.5 \mathrm{~mm}$. The rim, the clearance region around the hole, ranges from 0 to $0.4 \mathrm{~mm}$. The active area can be enlarged theoretically from few squared centimeters, as in the case of the prototypes used for the studies presented here, without limitations. These detectors can

\footnotetext{
* Corresponding author.

E-mail address: Stefano.Levorato@ts.infn.it (S. Levorato).
}

stand high rates up to $10 \mathrm{MHz} / \mathrm{mm}^{2}$, provide fast signals and reach high gains up to $10^{6}$ and more in triple stack configuration when detecting UV light. Due to the production technology the material budget is not particularly low and they do not offer a space resolution as good as GEMs. These aspects are not a limitation when detecting single photons in Cherenkov imaging counters. A THGEM-based photon detector usually consists of a structure of triple THGEM layers (see Fig. 1), where the first one, coated with a CsI film, acts as reflective photo-cathode $[3,4]$. The electric fields between the drift wires and the top face of the first THGEM (the photo-cathode), between two THGEM layers and between the third THGEM bottom face and the read out anode are indicated as drift field, transfer field and induction field, respectively.

The reader is referred to Refs. [5,6] for a detailed description of the tests performed to study the role played by the different geometrical parameters characterizing the THGEMs, for the 
production technology method chosen and for the discussion of the obtained result.

The detector optimization studies, measurement and test are the main topics in this paper.

\section{Gain issues and thickness role}

As reported in Refs. [3-6] high gains larger than $10^{5}$ are reachable with single or cascaded CsI-THGEM electrodes. When single photons are detected, the corresponding single photonelectron amplitude spectrum is exponentially distributed. Good signal to background ratio and a good gain stability of the detector are mandatory requirements in order that the threshold setting does not result in a critical issue.

In particular, large gains can be obtained multiplying electrons by THGEM with large rims but the gain stability versus time strongly depends on the rim size. Gain variations below 20\% are observed when the rim is not present while huge ones (up to a factor of 5 and more) are visible when the rim is large [5].

A possible way to overcome the gain instabilities due to large rim, preserving the high gain performance, is to increase the detector thickness and avoid the use of rim. The maximum gain achievable using a single THGEM stage for different gas mixtures and geometries is shown in Fig. 2. Details of the maximum gain test are described in Ref. [5]. The maximum gain obtained with

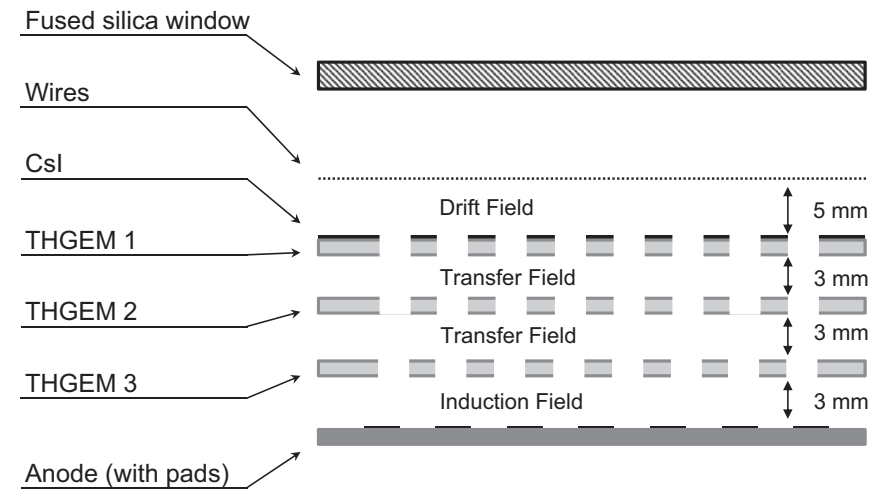

Fig. 1. Structure of a THGEM-based photon detector: the first layer is coated with a CSI photon converting film (not to scale). the $20 \mu \mathrm{m}$ rim, $0.4 \mathrm{~mm}$ thickness is recovered using a THGEM with no rim and double thickness. The hole diameter and pitch is the same for the three THGEMs tested.

\section{Photo-electron extraction from the CsI layer}

The effective photon detection efficiency of a THGEM based photon detector strongly depends, among other parameters, on the photo-electron extraction and on the subsequent photoelectron collection efficiency [8]. The last was studied in the present work both via electrostatic simulations and dedicated tests: the most favorable condition requires zero drift field as described in Ref. [5] and will not be discussed furthermore here.

The electric field on the photo-cathode surface, orthogonal to the THGEM surface $\left(E_{z}\right)$, generated by the dipole field of the THGEM holes must be large enough to ensure an effective photoelectron extraction. The values of $E_{z}$ for which the effective extraction efficiency is greater than $85 \%$ depend on the gas mixture used and correspond to values greater than $500 \mathrm{~V} / \mathrm{cm}$ in pure methane gas [8]. The same effective extraction is ensured by an $\mathrm{Ar}-\mathrm{CH}_{4}$ mixture with methane fraction larger than $30 \%$.

The layer where the CSI film is deposited can be further optimized by choosing an ad hoc geometry, as explained in the next lines, which is favorable to achieve high values of $E_{z}$ also in the farthest area from the holes' center, the center of the triangle having as vertexes' three holes' center (critical point). The $z$ component of the electric field, normal to the surface and in the critical point $\left(E_{z c}\right)$, is simulated with COMSOL Multiphysics ${ }^{\circledR}[9]$, as a function of the hole diameter $d$, for different values of the pitch $p$, keeping the $\Delta V$ applied to the THGEM electrodes fixed. The competing requirements of keeping an active surface area larger than $80 \%$ and a value of $\left|E_{z}\right|$ larger than $\sim 0.6 \mathrm{kV} / \mathrm{cm}$ bound the ratio of diameter and pitch to be $\sim 1 / 2$. Simulations, when fixing $d / p=\sim 1 / 2$, also show that the $E_{z c}$ component increases decreasing the THGEM thickness, i.e. allowing the dipole field of the THGEM hole to extend more outside the near hole region. Different configurations of the $d / p$ ratio result in an abrupt decrease of $E_{z c}$ : for a diameter and thickness of $400 \mu \mathrm{m}$ a change in pitch from 600 to $700 \mu \mathrm{m}$ results in a decrease from $\sim 0.8 \mathrm{kV} / \mathrm{cm}$ to $\sim 0.4 \mathrm{kV} / \mathrm{cm}$ for $E_{z c}$ when applying a $\Delta V=1.5 \mathrm{kV}$. These results point towards the use of a less thick first stage THGEM electrode with respect to the next ones where the parameter to be optimized is gain.
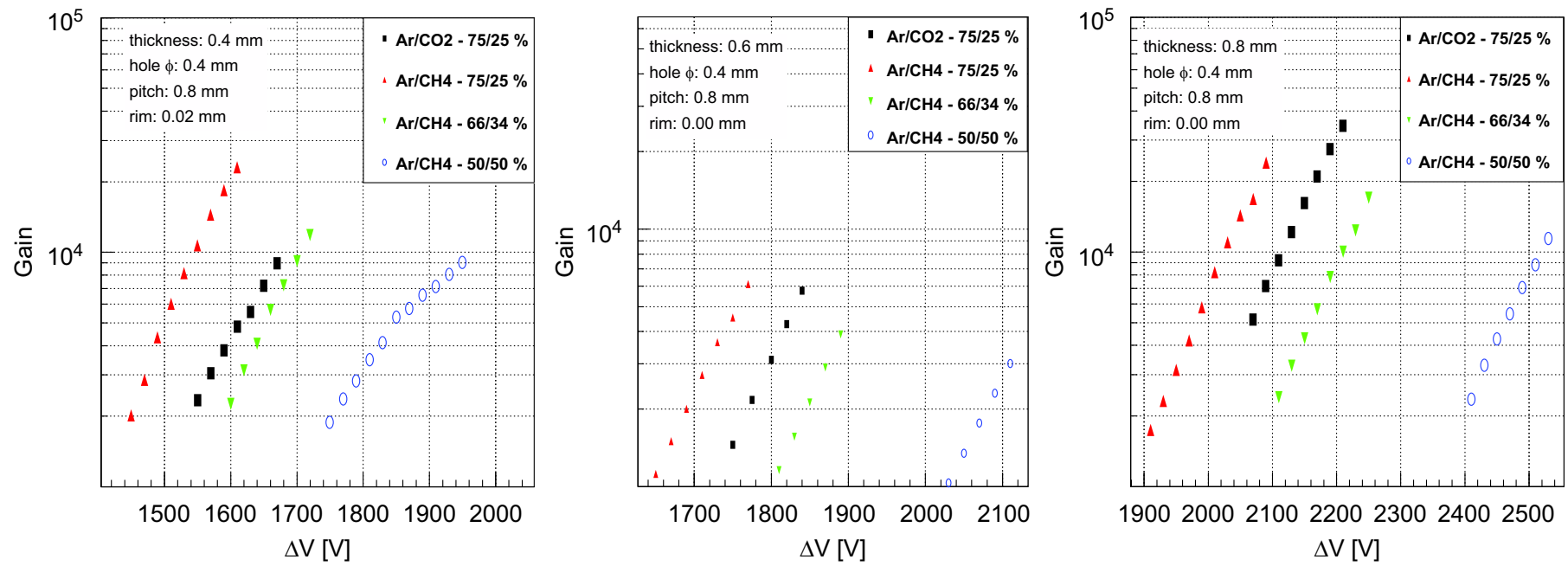

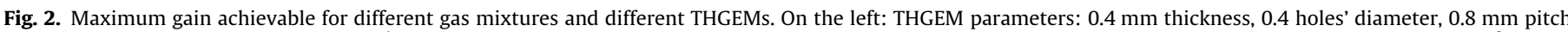

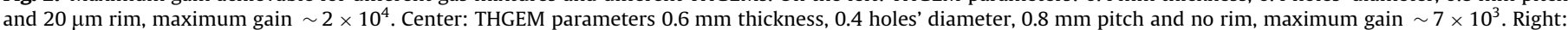
THGEM parameters $0.8 \mathrm{~mm}$ thickness, 0.4 holes' diameter, $0.8 \mathrm{~mm}$ pitch and no rim, maximum gain $\sim 2 \times 10^{4}$. 


\section{Time distribution spectra and photo-electron extraction efficiency}

During the 2010 test beam a multiple THGEM detector has been operated at the $\mathrm{H} 4150 \mathrm{GeV} / c \pi^{+}$line at CERN. It consists of three triple THGEM with $0.4 \mathrm{~mm}$ thickness, $0.4 \mathrm{~mm}$ holes diameter and $0.8 \mathrm{~mm}$ pitch $30 \mathrm{~mm} \times 30 \mathrm{~mm}$ active surface operated in $\mathrm{Ar} / \mathrm{CH}_{4} 50 / 50 \%$ mixtures at typical gain of $10^{5}$ and a Hamamatsu MAPMT R7600 [12,13]. The THGEMs are powered via resistive divider generating the required electric fields $[5,6]$. The four detectors (see Fig. 3) are arranged to intercept four arcs of the focused Cherenkov light emitted by the hemispherical quartz radiator traversed by beam particles. The trigger system consists of scintillator signal coincidences ensuring that a beam particle has crossed the radiator and the read out electronic is based on the CMAD front end chip and the F1 TDC chip used for the COMPASS experiment $[10,11]$. In central part of Fig. 3 , it is possible to observe the pileup of events collected with the two lateral THGEM detectors: the intercepted corona sectors are clearly evident. In Fig. 3 right, the timing distribution of the THGEMs and MAPMT response are shown. The time delay between the signals by the MAPMT and the THGEMs is of the order of $130 \mathrm{~ns}$. The electron speed in $\mathrm{Ar} / \mathrm{CH}_{4} 50 / 50 \%$ in a field of $1.5 \mathrm{kV} / \mathrm{cm}$ is $\sim 8 \times 10^{6} \mathrm{~cm} / \mathrm{s}$. The expected transit time for $1 \mathrm{~cm}$ path, namely the THGEM detector vertical size, is $\sim 125$ ns to be compared with the measured time difference.
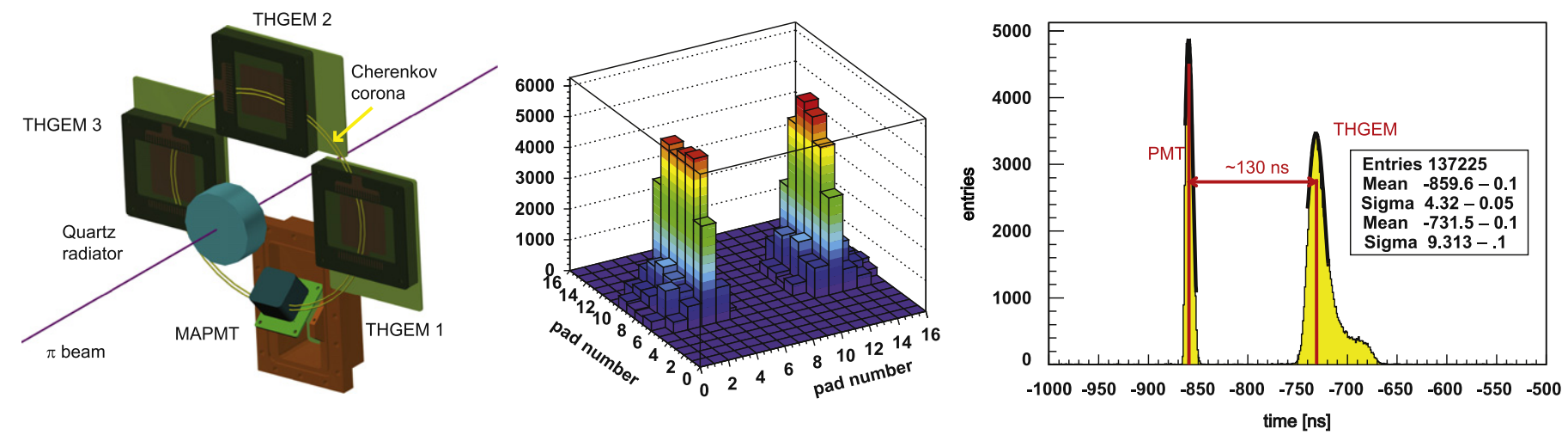

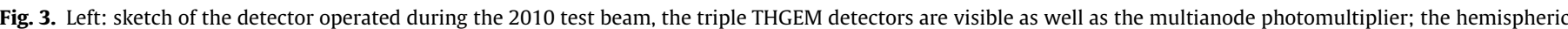

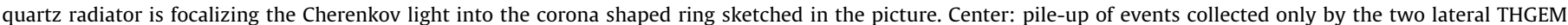

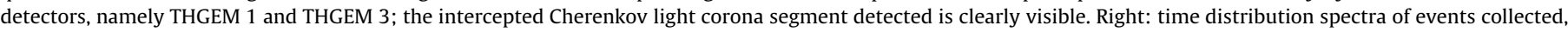
the first peak is the MAPMT response while the second one, 130 ns later, comes from THGEM response.
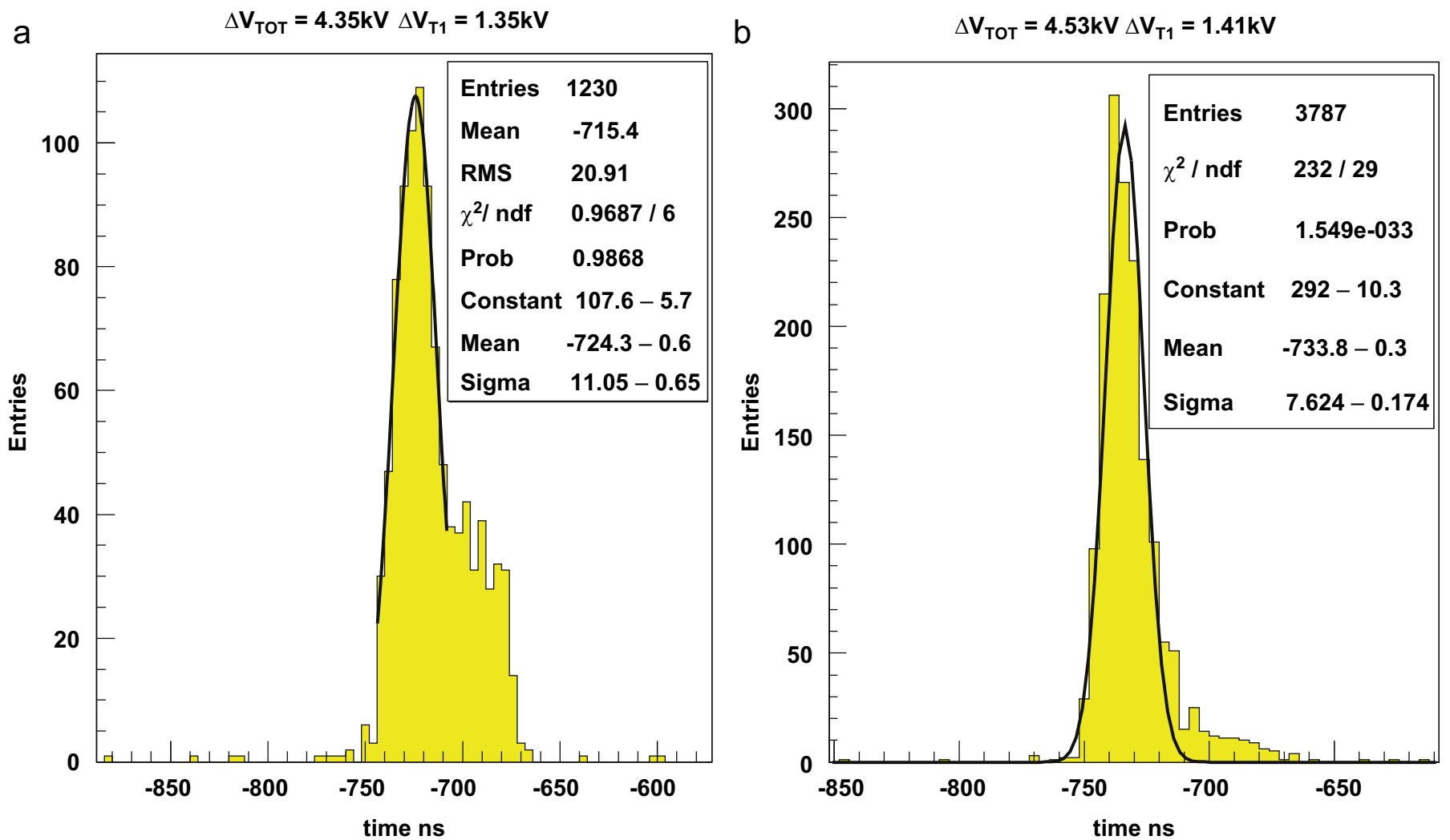

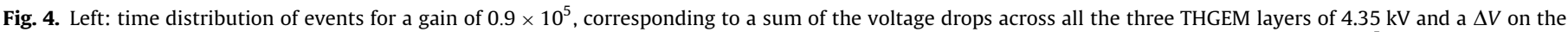

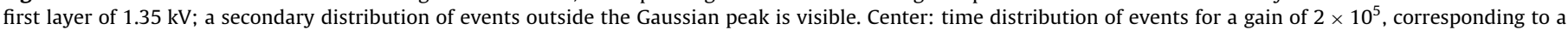
sum of the voltage drops across all the three THGEM layers of $4.53 \mathrm{kV}$; the secondary peak is reduced. 
The typical time spectra collected are shown in Fig. 4(a,b) for two different values of the total gain $0.9 \times 10^{5}$ and $2.0 \times 10^{5}$ corresponding, respectively, to a sum of the $\Delta V_{i}$ applied to the THGEM electrodes of 4.35 and $4.53 \mathrm{kV}$, and a $\Delta V$ across the first THGEM electrodes of $1.35 \mathrm{kV}$ and $1.41 \mathrm{kV}$, respectively. The two timing spectra present a main component with a $\sigma$ of 11.1 and $7.6 \mathrm{~ns}$. The population of the secondary component, delayed of nearly 20 ns and corresponding to $23 \%$ and to $6 \%$ of the events outside the Gaussian distributed peak, decreases with the gain increase i.e. with the increase of the $\Delta V$ applied to the THGEM layers. As indicated by simulations for our geometrical choice of the parameters the field on the first THGEM CsI surface is $\sim 500 \mathrm{~V} / \mathrm{cm}$ in the critical point and it increases moving along the test line, in absolute value of $50 \mathrm{~V}$ per $100 \mu \mathrm{m}$. If this value is compared with the electron speed in $\mathrm{Ar}$ and in $\mathrm{CH}_{4}$ gas [7] field variation, the photoelectron experiences before entering the hole are responsible for delays of several ns, comparable with the observed, in the timing distribution between events generated by photoelectrons converted near the holes (few $\mu \mathrm{m}$ ) and near the critical point. The timing distribution spectrum when combined with photoelectron extraction curves as in Ref. [8] thus becomes a valid method to check if the field conditions on the whole surface guarantee full photoelectron extraction efficiency and results helpful in guiding the choice of the parameters for the first THGEM layer. The consistency of the measured timing variation in the spectra for the different gain conditions i.e. field conditions and the expected ones suggested by simulations also becomes a proof of the reliability for the electrostatic simulation results.

\section{Detector optimization issue}

As discussed in the previous sections the different THGEM electrodes require different geometrical parameter choices according to the function they are assigned. The studies performed suggest the use of a thinner THGEM layer as reflective photo-cathode: this choice in fact allows for the maximization of the vertical component of the electric field on the CsI surface and consequently of the photo-electron extraction efficiency, as explained at the end of Section 4. Thicker layers can be used for the 2 nd and 3rd THGEM multiplication stages. The two competing requirements, the maximization of the photon detection efficiency and of the photo-sensitive active area, bound the ratio $d / p=\sim 1 / 2$, leaving thickness as the only free parameter to increase the $E_{z}$ field at the photo-sensitive surface. Moreover while the optimization of one layer for a specific task is easily accomplished, when the layers are put together their interplay is not negligible requiring an iterative steps' procedure to approach the full optimization. The not yet completely solved problem of the fraction of ions, flowing back to the photo-cathode surface and generating instabilities, probably requires a further optimized layer to reach a stable operation of the detector at high gains.

At the time of this talk larger size detectors, $300 \mathrm{~mm} \times$ $300 \mathrm{~mm}$, are under assembling operation and will be tested during 2011 and 2012 test beams.

\section{Acknowledgments}

K. Novakova and L. Steiger are supported by the Student Grant SGS 2011/7821 - Interactive mechatronic systems in computer engineering.

\section{References}

[1] L. Periale, et al., Nuclear Instruments and Methods in Physics Research Section A 478 (2002) 377.

[2] A. Breskin, et al., Nuclear Instruments and Methods in Physics Research Section A 598 (2009) 107 and references therein.

[3] V. Peskov, et al., Journal of Instrumentation 5 (2010) P11004.

[4] P. Martinengo, et al., Nuclear Instruments and Methods in Physics Research Section A 639 (2011) 126.

[5] M. Alexeev, et al., Journal of Instrumentation 5 (2010) P03009.

[6] M. Alexeev, et al., Nuclear Instruments and Methods in Physics Research Section A 639 (2010) 130.

[7] F. Sauli, 77-09, fig. 26 pg. 29, 3 May 1977.

[8] C.D.R. Azevedo, et al., Journal of Instrumentation 5 (2010) P01002.

[9] COMSOL, Inc. Palo Alto, 〈http://www.comsol.com >.

[10] P. Abbon, et al., Nuclear Instruments and Methods in Physics Research Section A 587 (2008) 371.

[11] H. Fischer, et al., Nuclear Instruments and Methods in Physics Research Section A 461 (2001) 507.

[12] 〈http://www.hamamatsu.com〉.

[13] P. Abbon, et al., Nuclear Instruments and Methods in Physics Research Section A 580 (2007) 906 